\title{
The impact of post-operative sepsis on mortality after hospital discharge among elective surgical patients: a population-based cohort study
}

Lixin $\mathrm{Ou}^{1,2^{*}}$ (D) Jack Chen ${ }^{1,2}$, Ken Hillman ${ }^{1,2}$, Arthas Flabouris ${ }^{3,4}$, Michael Parr ${ }^{5}$, Hassan Assareh ${ }^{6}$ and Rinaldo Bellomo ${ }^{7}$

\begin{abstract}
Background: Our aim in the present study was to assess the mortality impact of hospital-acquired post-operative sepsis up to 1 year after hospital discharge among adult non-short-stay elective surgical patients.

Methods: We conducted a population-based, retrospective cohort study of all elective surgical patients admitted to 82 public acute hospitals between 1 January 2007 and 31 December 2012 in New South Wales, Australia. All adult elective surgical admission patients who stayed in hospital for $\geq 4$ days and survived to discharge after post-operative sepsis were identified using the Admitted Patient Data Collection records linked with the Registry of Births, Deaths, and Marriages. We assessed post-discharge mortality rates at 30 days, 60 days, 90 days and 1 year and compared them with those of patients without post-operative sepsis.

Results: We studied 144,503 survivors to discharge. Of these, 1857 (1.3\%) had experienced post-operative sepsis. Their post-discharge mortality rates at 30 days, 60 days, 90 days and 1 year were $4.6 \%, 6.7 \%, 8.1 \%$ and $13.5 \%$ (vs $0.7 \%, 1.2 \%, 1.5 \%$ and $3.8 \%$ in the non-sepsis cohort), respectively $(P<0.0001$ for all). After adjustment for patient and hospital characteristics, post-operative sepsis remained independently associated with a higher mortality risk (30-day mortality HR 2.75, 95\% Cl 2.14-3.53; 60-day mortality HR 2.45, 95\% Cl 1.94-3.10; 90-day mortality HR 2.31, 95\% Cl 1.85-2.87; 1-year mortality HR 1.71, 95\% Cl 1.46-2.00). Being older than 75 years of age (HR 3.50, 95\% Cl 1.56-7.87) and presence of severe/very severe co-morbidities as defined by Charlson co-morbidity index (severe vs normal HR 2.05, 95\% Cl 1.45-2.89; very severe vs normal HR 2.17, 95\% Cl 1.49-3.17) were the only other significant independent predictors of increased 1-year mortality.

Conclusions: Among elective surgical patients, post-operative sepsis is independently associated with increased post-discharge mortality up to 1 year after hospital discharge. This risk is particularly high in the first month, in older age patients and in the presence of severe/very severe co-morbidities. This high-risk population can be targeted for interventions.
\end{abstract}

\footnotetext{
* Correspondence: lixin.ou@unsw.edu.au

${ }^{1}$ Simpson Centre for Health Services Research, South Western Sydney Clinical

School, University of New South Wales, Sydney, New South Wales, Australia

${ }^{2}$ Ingham Institute for Applied Medical Research, Liverpool, New South Wales,

Australia

Full list of author information is available at the end of the article
} 


\section{Background}

Post-operative sepsis is a leading cause of multiple organ dysfunction and in-hospital mortality [1-3]. The U.S. Centers for Disease Control and Prevention reported that about 1 in 25 patients experience at least one healthcare-associated infection during hospitalisation [4]. Patients who are admitted with or who develop sepsis in hospital also have an increased risk of death following hospital discharge $[5,6]$. Such sepsis-associated risk of death is higher than in the general population and remains higher for up to 5 years following hospital discharge [7, 8]. Moreover, long-term healthcare costs for sepsis survivors are higher. Researchers in a recent study reported that $42.7 \%$ of severe sepsis survivors were re-hospitalized within 90 days [9], incurring higher costs, especially in the first year after hospital discharge, when costs are approximately three times the costs in the following 2-3 years [10]. Given such high prevalence, significant risk of mortality, poor prognosis and high healthcare resource consumption, the U.S. Agency for Healthcare Research and Quality (AHRQ) has proposed 'post-operative sepsis' as a key patient safety indicator, aiming to monitor potentially preventable surgical complications among elective surgical patients without serious medical conditions at admission [11]. Thus, the indicator 'post-operative sepsis' was developed after a comprehensive literature review; analysis of International Classification of Diseases, Ninth Revision, Clinical Modification (ICD-9-CM), codes; review by a clinician panel; implementation of risk adjustment; and performing empirical analyses. This quality indicator has been widely used in the United States to measure aspects of patient safety and quality and to monitor the impact of quality improvement initiatives [3, 11-14].

Despite the importance of sepsis, most studies in which investigators have examined the long-term outcomes of sepsis survivors have been limited to a single institution [10,15-17], or to patients specifically admitted via emergency rooms [18], or to intensive care units (ICUs) [7, 19, 20]. Most of these long-term outcome studies have included all sources of sepsis, such as a mix of community- or hospital-acquired sepsis, as well as a mix of medical and surgical patients [5]. In contrast, the few studies in which researchers have examined 30-day or 1-year post-discharge mortality among elective surgical patients with post-operative sepsis have narrowly targeted specific patient groups (e.g., patients with cancer or elderly patients aged $\geq 65$ years) and surgery types (e.g., digestive or abdominal aortic aneurysm [AAA] surgery), limiting their generalisability $[21,22]$.

Accordingly, we conducted a population-based study of long-term mortality among patients with post-operative sepsis up to 1 year after discharge, and we compared postdischarge mortality and its associated risk factors with those of elective surgical patients without post-operative sepsis admitted to all public acute hospitals in the state of New South Wales (NSW), Australia. We targeted those patients who stayed in hospital beyond 3 days and survived to discharge, and we aimed to test the hypothesis that patients with post-operative sepsis who are discharged alive from hospital have a higher risk of death, even at 1 year after their index admission. We also explored the hypothesis that post-operative sepsis in these patients is an independent predictor of death and that other risk factors can be identified that are associated with such increased risk.

\section{Methods}

\section{Data source and study population}

We performed a population-based retrospective cohort study using NSW administrative data derived from the Admitted Patient Data Collection (APDC) records. The APDC includes information on patient demographics, medical conditions and procedures, hospital characteristics, and separations (discharges, transfers and deaths) from all hospitals in NSW. The medical records for each episode of care in the APDC were assigned codes based on the International Statistical Classification of Diseases and Related Health Problems, Tenth Revision, Australian Modification (ICD-10-AM) [23]. Each hospital has certified and trained coders who follow standardised procedures to generate these codes from information in medical records.

The study included adult elective surgical patients who were admitted to $82 \mathrm{NSW}$ public acute hospitals between 1 January 2007 and 31 December 2012 and survived to discharge. We identified our study population on the basis of selection criteria developed by the AHRQ for postoperative sepsis. According to the AHRQ selection criteria, we included all elective surgical patients (aged $\geq 18$ years) who had any primarily performed procedures with operating room procedure codes and not admitted through the emergency department and who did not have a short hospital stay (Fig. 1). We excluded those patients who fulfilled any one of the following exclusion criteria:

- A principal diagnosis on admission that was sepsis or infection (see Additional file 1: Appendix 1; Additional file 2: Appendix 2)

- Any ICD-10-AM diagnosis codes for cancer (see Additional file 2: Appendix 2)

- Any ICD-10-AM diagnosis codes or any ICD-10-AM procedure codes for immunocompromised state (see Additional file 2: Appendix 2)

- Major Diagnostic Categories 14 (pregnancy, childbirth and puerperium)

- A stay in hospital of less than 4 days

- Missing data on discharge status, sex, age, year or principal diagnosis (There were no missing values for these variables among the elective surgical admissions aged $\geq 18$ years included in our study.) 


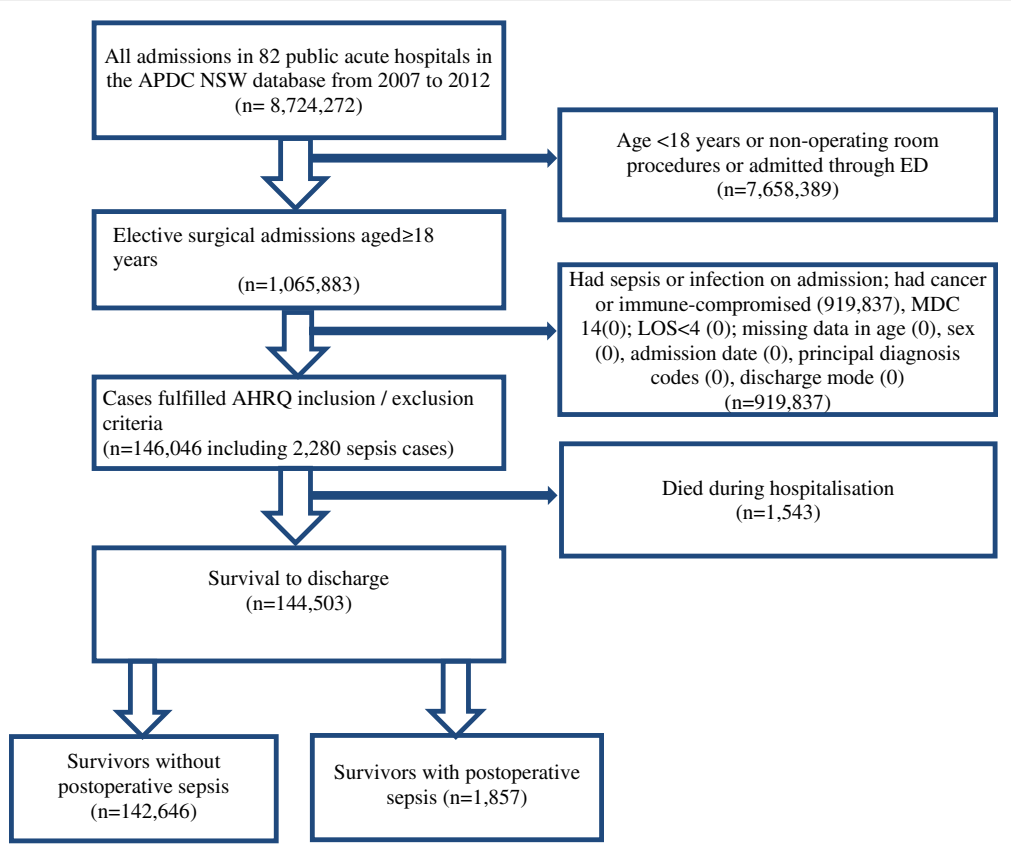

Fig. 1 Flowchart illustrating the derivation of the study population. AHRQ Agency for Healthcare Research and Quality, APDC Admitted Patient Data Collection, ED Emergency department, LOS Length of stay, MDC Major Diagnostic Categories

Among the selected population, cases with postoperative sepsis were identified by ICD-10-AM diagnosis codes (see Additional file 1: Appendix 1). Because of the difference in coding systems used in the United States (ICD-9-CM) and Australia (ICD-10-AM), all diagnoses and procedure codes in the AHRQ definition were translated to ICD-10-AM codes according to the Organisation for Economic Co-operation and Development (OECD) technical manual for patient safety indicators [24]. We derived outcome variables of post-operative sepsis using 54 non-principal diagnostic fields in the medical record by ICD-10-AM codes matched from the OECD manual (see Additional file 1: Appendix 1). We then classified study population into sepsis and non-sepsis cohorts (Fig. 1).

The selected data were linked to the NSW Registry of Births, Deaths, and Marriages (RBDM) through the Centre of Health Record Linkage, NSW Ministry of Health, to derive survival status within 1 year after discharge. We excluded those patients whose survival status was not available up to 1 year after discharge because the RBDM data obtained in our study spanned only from 1 January 2007 to 28 March 2014. As a result, the study period was presented as admission years between 2007 and 2012 .

\section{Patient demographic and hospital characteristics}

Patient demographic information included age, sex, country of birth, marital status, severity of illness/co-morbidity, and advantaged and disadvantaged Socio-Economic
Indexes for Areas (SEIFA) scores [25] (categorised into four classes, from first quartile $=$ most disadvantaged areas to fourth quartile $=$ most advantaged areas) representing patient socio-economic status. Severity of illness/ co-morbidity was defined by the Charlson co-morbidity index score based on the ICD-10 coding scheme [26]. We classified the severity of illness/co-morbidity into four categories: normal (index score $=0$ ), moderate (index score $=1$ ), severe (index score $=2$ ) and very severe (index score $\geq 3$ ). Because the AHRQ selection criteria excluded patients with any cancer or immunocompromised state, three of the Charlson co-morbidities-any malignancy (including leukaemia and lymphoma), metastatic solid tumour and AIDS/HIV-were excluded. Thus, the comorbidities in this study encompassed only 14 of the Charlson co-morbidity index-defined co-morbidities: myocardial infarction, congestive heart failure, peripheral vascular disease, cerebrovascular disease, dementia, chronic pulmonary disease, rheumatologic disease, peptic ulcer disease, mild liver disease, diabetes without chronic complications, diabetes with chronic complications, hemiplegia or paraplegia, renal disease, and moderate or severe liver disease.

Hospital characteristics, including the location (metropolitan, rural and regional NSW) as well as peer group classification: (A1 = principal referral, usually teaching hospitals; A3 = ungrouped acute; $\mathrm{B}=$ major metropolitan and non-metropolitan; $\mathrm{C} 1=$ district group 1 ; $\mathrm{C} 2=$ district group 2). Peer hospital groups were divided into 
those of similar type and size, ranging from treating 25,000 or more acute case-mix-weighted separations per annum in the principal referral group through to treating $\geq 2000$ or more (but $<5000$ ) acute case-mixweighted separations per annum in district group 2 [27]. Using appropriate procedure codes from ICD-10AM (Additional file 3: Appendix 3) [24], we defined six groups of major surgical procedures: coronary artery bypass graft (CABG), abdominal surgery, endovascular aneurysm repair, total hip replacement, total knee replacement and other surgical procedures.

\section{Study outcomes}

The primary outcome was post-hospital discharge mortality at 30 days, 60 days, 90 days and 1 year. We used the date of death and the date of discharge to define the length of survival. Post-discharge mortality in the sepsis cases was calculated as the number of deaths in the group at 30 days, 60 days, 90 days and 1 year after the discharge date divided by the number of sepsis cases, respectively. A similar calculation of post-discharge mortality was performed in the non-sepsis group.

\section{Statistical analysis}

Baseline characteristics were compared between the non-sepsis and sepsis cohorts by unpaired $t$ test and the Rao-Scott chi-square test. To understand changes in 1year mortality over time, we assessed the crude linear trend for the outcome variables after excluding a possible quadratic effect using the study year as a continuous variable and employing Poisson mixed models. Cumulative survival outcomes across the non-sepsis and sepsis cohorts were estimated first by calculating the Kaplan-Meier survival functions and using log-rank tests to detect differences between the two cohorts up to 1 year after discharge for differences in age-, sex- and co-morbidity-specific mortality rates in the two cohorts. We then estimated the risk difference of death at 30 days, 60 days, 90 days and 1 year between the two cohorts using Cox proportional hazards models to control for the other confounding factors, which included patient demographics (baseline age, sex, country of birth, marital status, severity of illness/co-morbidity and SEIFA score), hospital characteristics (location and peer groups), major surgical procedures, length of hospital stay and the year of admission.

We conducted a sensitivity analysis using 1:1 nearestneighbour matching (NNM) (based on Euclidean matrix) as recommended on the basis of recent research [28], in comparison with the popular propensity score matching (PSM) (1:1; based on logistic regression). For NNM, the matching was based on admission year, age, sex, country of birth, marital status, severity of illness/co-morbidity, socio-economic status (quartile of SEIFA score), location of the hospital (rural/regional vs others), hospital peer group, surgery type and length of stay. (The exact matching was done on the basis of age group, country of birth and co-morbidity.) The estimation of treatment effect was based on robust variance estimator and adjusted for age and length of stay. For PSM, the same matching variables were used, except that there were no exact matching groups and no adjustment for the continuous variables of age and length of stay. For both NNM and PSM, a caliper of 0.05 was employed, and a check for overlap of both baseline distribution and matched samples was done. Both graphic plots and standardised statistical summary and tests were employed wherever appropriate to ensure the balance of matched samples. We present the results from both NNM and PSM in Additional file 4: Appendix 4.

We took into account hospital cluster effect within these models using a robust cluster variance estimator. The risk of death is presented as an HR, and 95\% CIs were calculated around the estimated HRs. A $P$ value of 0.05 was considered as indicative of statistical significance for sepsis and non-sepsis cohorts, and 95\% CIs are presented where appropriate. All analyses were performed using STATA 14 software (StataCorp, College Station, TX, USA). This study was approved by the NSW Population \& Health Services Research Ethics Committee.

\section{Results}

A total of 146,046 elective surgical admission patients between 2007 and 2012 fulfilled the study selection criteria, and 2279 (15.6 per 1000 cases) developed postoperative sepsis. Of the 144,503 patients who survived to hospital discharge, 1857 (12.9 per 1000 cases) were hospital survivors after post-operative sepsis (Fig. 1, Table 1). The number of elective surgical patients who survived to discharge was similar in each admission year. However, the incidence of post-operative sepsis increased over time, whereas both the overall mortality rate and the mortality rate of those with sepsis declined (Fig. 2) over time. Accordingly, the proportion of post-operative sepsis survivors among all elective surgical patients increased from 11.8 per 1000 cases in 2007 to 15.5 per 1000 cases in 2012 (31.4\% increase; $P<0.001$ for trend) (Fig. 2). Compared with the non-sepsis cohort, patients with sepsis were older, more likely to be male, more likely to have severe/very severe illness, and more likely to be admitted to a hospital in a metropolitan area or to a principal referral hospital (Table 1). Almost half of the patients in the sepsis group and one-third in the nonsepsis group underwent CABG or abdominal surgery. The length of hospital stay for patients with sepsis was much longer than that of the non-septic patients (33.4 days vs 9.1 days on average, $P<0.001$ ). 
Table 1 Baseline patient demographic and hospital characteristics according to presence or absence of post-operative sepsis ${ }^{\mathrm{a}}$ $(n=144,503)$

\begin{tabular}{|c|c|c|c|}
\hline Characteristics & Non-sepsis & Sepsis & IR of sepsis \\
\hline Total & 142,646 & 1857 & 12.9 \\
\hline \multicolumn{4}{|l|}{ Year of admission } \\
\hline 2007 & $23,564(16.5 \%)$ & $281(15.1 \%)$ & 11.8 \\
\hline 2008 & $23,993(16.8 \%)$ & $293(15.8 \%)$ & 12.1 \\
\hline 2009 & $23,305(16.3 \%)$ & $280(15.1 \%)$ & 11.9 \\
\hline 2010 & $24,447(17.1 \%)$ & $324(17.5 \%)$ & 13.1 \\
\hline 2011 & 24,195 (17.0\%) & 315 (17.0\%) & 12.9 \\
\hline 2012 & $23,142(16.2 \%)$ & $364(19.6 \%)$ & 15.5 \\
\hline \multicolumn{4}{|l|}{ Hospitalisation in preceding year } \\
\hline 2007 & N/A & N/A & \\
\hline 2008 & $2275 / 23,993(9.5 \%)$ & $35 / 293(12.0 \%)$ & \\
\hline 2009 & 2734/23,305 (11.7\%) & $40 / 280(14.3 \%)$ & \\
\hline 2010 & $3344 / 24,447$ (13.7\%) & $46 / 324(14.2 \%)$ & \\
\hline 2011 & $3771 / 24,195(15.6 \%)$ & $54 / 315(17.1 \%)$ & \\
\hline 2012 & $3889 / 23,142(16.8 \%)$ & $64 / 364(17.6 \%)$ & \\
\hline \multicolumn{4}{|l|}{ Age } \\
\hline$\geq 18$ years to $<35$ years & $8838(6.2 \%)$ & $106(5.7 \%)$ & 11.9 \\
\hline$\geq 35$ years to $<55$ years & $28,015(19.6 \%)$ & $348(18.7 \%)$ & 12.3 \\
\hline$\geq 55$ years to $<75$ years & $64,382(45.1 \%)$ & $814(43.8 \%)$ & 12.5 \\
\hline$\geq 75$ years & $41,411(29.0 \%)$ & $589(31.7 \%)^{*}$ & 14 \\
\hline Mean \pm SD & $63.8 \pm 16.3$ & $64.8 \pm 16.3^{* *}$ & \\
\hline \multicolumn{4}{|l|}{ Sex } \\
\hline Male & $67,011(47.0 \%)$ & $1147(61.8 \%)^{* *}$ & 16.8 \\
\hline Female & $75,635(53.0 \%)$ & $710(38.2 \%)^{* *}$ & 9.3 \\
\hline \multicolumn{4}{|l|}{ Country of birth } \\
\hline Australia and New Zealand & $98,787(69.3 \%)$ & $1249(67.3 \%)$ & 12.5 \\
\hline United Kingdom, United States and Canada & $10,205(7.2 \%)$ & $111(6.0 \%)^{*}$ & 10.8 \\
\hline Non-English-speaking Europe & $15,949(11.2 \%)$ & $220(11.8 \%)$ & 13.6 \\
\hline North Africa & $5564(3.9 \%)$ & $78(4.2 \%)$ & 13.8 \\
\hline Asia & $6,922(4.9 \%)$ & $93(5.0 \%)$ & 13.3 \\
\hline Others & $4,222(3.0 \%)$ & $77(4.1 \%)^{* *}$ & 17.9 \\
\hline Unknown & $997(0.7 \%)$ & $29(1.6 \%)^{* *}$ & 28.3 \\
\hline \multicolumn{4}{|l|}{ Marital status } \\
\hline Married & $77,810(54.6 \%)$ & $1005(54.1 \%)$ & 12.8 \\
\hline Single & $62,745(44.0 \%)$ & $805(43.3 \%)$ & 12.7 \\
\hline Unknown & $1923(1.3 \%)$ & $47(2.5 \%)^{* *}$ & 23.9 \\
\hline \multicolumn{4}{|l|}{ Severity of illness/co-morbidity } \\
\hline Normal & $116,031(81.3 \%)$ & $969(52.2 \%)^{* *}$ & 8.3 \\
\hline Moderate & 16,509 (11.6\%) & $322(17.3 \%)^{* *}$ & 19.1 \\
\hline Severe & $5700(4.0 \%)$ & $213(11.5 \%)^{* *}$ & 36.0 \\
\hline Very severe & 4406 (3.1\%) & $353(19.0 \%)^{* *}$ & 74.2 \\
\hline
\end{tabular}


Table 1 Baseline patient demographic and hospital characteristics according to presence or absence of post-operative sepsis ${ }^{\mathrm{a}}$ $(n=144,503)$ (Continued)

\begin{tabular}{|c|c|c|c|}
\hline \multicolumn{4}{|l|}{ SEIFA scores } \\
\hline First quartile (most disadvantaged) & $48,634(34.1 \%)$ & $590(31.8 \%)^{*}$ & 12 \\
\hline Second quartile & $41,195(28.9 \%)$ & $568(30.6 \%)$ & 13.6 \\
\hline Third quartile & $31,862(22.3 \%)$ & $408(22.0 \%)$ & 12.6 \\
\hline Fourth quartile (most advantaged) & $19,888(13.9 \%)$ & $267(14.4 \%)$ & 13.2 \\
\hline Unknown & $1067(0.7 \%)$ & $24(1.3 \%)^{* *}$ & 22 \\
\hline \multicolumn{4}{|l|}{ Local health district of facilities } \\
\hline Metropolitan & $91,018(63.8 \%)$ & $1336(71.9 \%)^{* *}$ & 14.5 \\
\hline Rural & $44,902(31.5 \%)$ & $420(22.6 \%)^{* *}$ & 9.3 \\
\hline Unknown & $6726(4.7 \%)$ & $101(5.4 \%)$ & 14.8 \\
\hline \multicolumn{4}{|l|}{ Peer hospital group } \\
\hline Principal referral & $85,108(59.7 \%)$ & $1358(73.1 \%)^{* *}$ & 15.7 \\
\hline Ungrouped acute & $2418(1.7 \%)$ & $6(0.3 \%)^{* *}$ & 2.5 \\
\hline Major metropolitan and non-metropolitan & $44,850(31.4 \%)$ & $444(23.9 \%)^{* *}$ & 9.8 \\
\hline District groups 1 and 2 & $10,270(7.2 \%)$ & $49(2.6 \%)^{* *}$ & 4.7 \\
\hline \multicolumn{4}{|l|}{ Major surgical procedure } \\
\hline CABG & $8994(6.3 \%)$ & $186(10.0 \%)^{* *}$ & 20.3 \\
\hline Abdominal surgery & $33,506(23.5 \%)$ & $649(35.0 \%)^{* *}$ & 19.0 \\
\hline EVAR & $1458(1.0 \%)$ & $20(1.1 \%)$ & 13.5 \\
\hline Total hip replacement & $13,632(9.6 \%)$ & $45(2.4 \%)^{* *}$ & 3.3 \\
\hline Total knee replacement & $22,598(15.8 \%)$ & $57(3.1 \%)^{* *}$ & 2.5 \\
\hline Other & $62,458(43.8 \%)$ & $900(48.5 \%)^{* *}$ & 14.2 \\
\hline \multicolumn{4}{|l|}{ Length of stay } \\
\hline Mean \pm SD & $9.1 \pm 9.6$ & $33.4 \pm 31.6$ & \\
\hline Median (IQR) & $6(5-10)$ & $24(14-42)$ & \\
\hline
\end{tabular}

\section{Mortality at 30 days, 60 days, 90 days and 1 year post-discharge}

The unadjusted post-discharge mortality rates at 30 days, 60 days, 90 days and 1 year in the sepsis cohort were $4.6 \%, 6.7 \%, 8.1 \%$ and $13.5 \%$, respectively (vs $0.7 \%$, $1.2 \%, 1.5 \%$ and $3.8 \%$ in the non-sepsis cohort, respectively; $P<0.0001$ ) (Table 2) with a proportionally strong increase in risk in the first 30 days.

Kaplan-Meier survival analysis confirmed a significantly reduced survival at 1 year post-discharge in patients with sepsis $(86.5 \%$ vs $96.2 \%$; $P<0.001$ by log-rank test) (Fig. 3 ), with the greatest rate of decline occurring within the first 30 days post-discharge. Although decreased survival in patients with sepsis was observed across all four age groups $(P<0.001)$ (Fig. 4), the effect was most pronounced among patients aged 75 years or older (Fig. 4d). After adjustment for patient and hospital characteristics, the risk of mortality was consistently higher at all time points in the sepsis cohort than in the non-sepsis cohort (Table 2). Both NNM and PSM approaches showed a consistent, elevated, significant risk of post-discharge mortality (at 30 days, 60 days and 1 year) (see Additional file 4: Appendix 4). These analyses showed that the absolute risk of death at 1 year post-discharge in the sepsis cohort was $5.4 \%$ higher than in the non-septic cohort.

\section{Multivariate analysis of 1-year mortality and its trends}

The 1-year post-discharge mortality did not change significantly over the study period (2007-2012) in the nonsepsis and sepsis cohorts (Table 3 ). The Cox proportional hazards models displayed different patterns of the risk factors predicting 1-year mortality in the two cohorts. In the non-sepsis cohort, individual factors, including advanced age, male sex, place of birth, marital status, hospital peer 


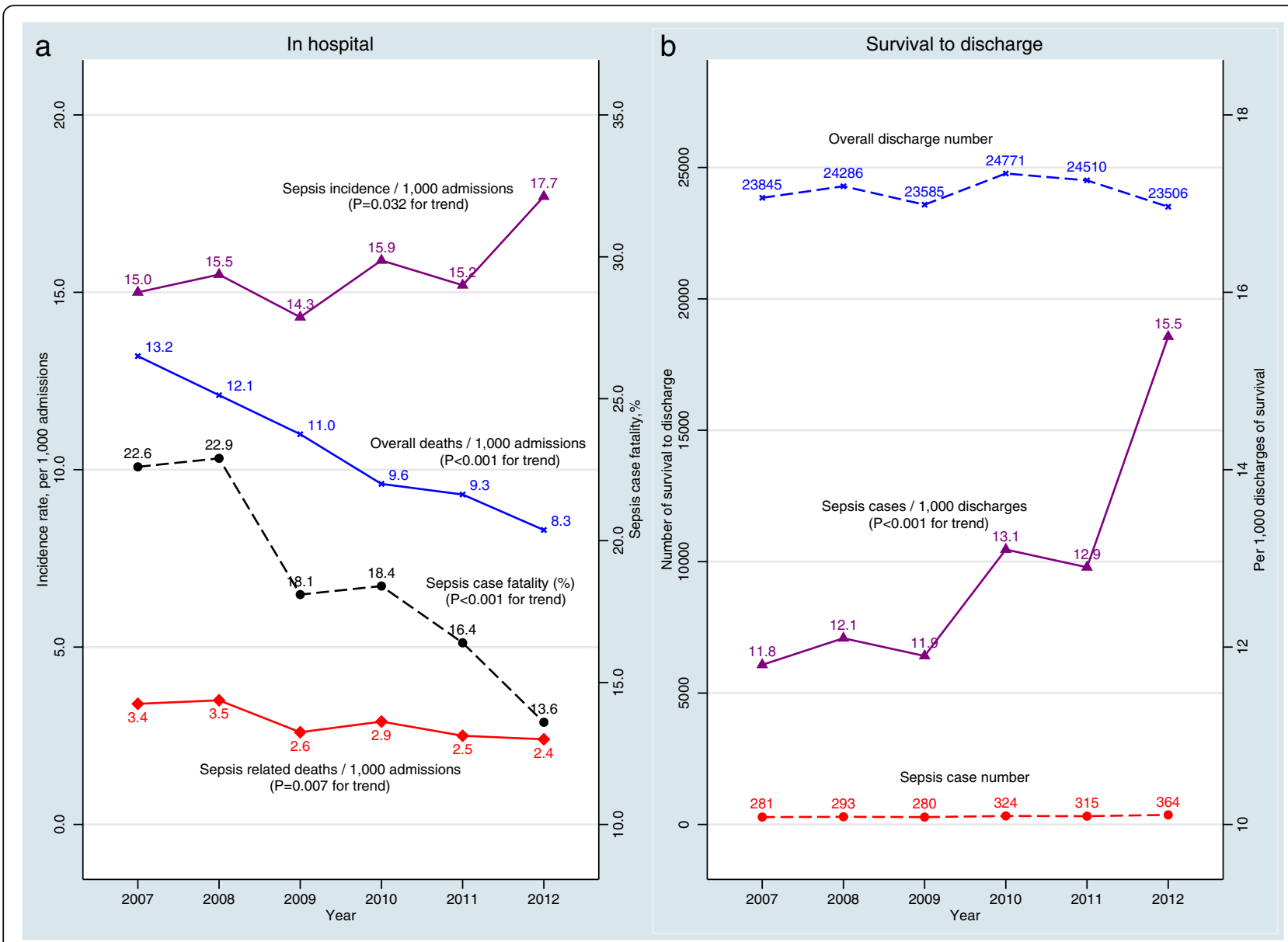

Fig. 2 Trends in the incidence rate of post-operative sepsis and sepsis-related mortality in hospitals (a) and the number of survival to discharge (b), 2007-2012

group, surgical type and number of co-morbidities, predicted increasing risk of death. In the sepsis cohort, only older age (75 years or older; HR 3.50, 95\% CI 1.56-7.87), hospital peer group, surgical type and pre-exiting severe/very severe co-morbidities (severe HR 2.05, 95\% CI 1.45-2.89; very severe HR 2.17, 95\% CI 1.49-3.17) carried an increased risk of death at 1 year after discharge. Older age and severity of illness/co-morbidity were two independent risk factors that increased the risk of death in both the sepsis and non-sepsis cohorts.

The magnitude of risk of age on 1-year post-discharge mortality in sepsis cohort (HR 3.50 for age $\geq 75$ years) was smaller than that in the non-sepsis cohort (HR 20.0). The risk of severity of illness/co-morbidity on death was also lower in the sepsis cohort (moderate vs normal HR 0.96, 95\% CI 0.61-1.50; non-significant)

Table 2 Post-discharge mortality and adjusted HRs at 30 days, 60 days, 90 days and 1 year between the two cohorts (pooled 2007-2012 data, $n=144,503)$

\begin{tabular}{|c|c|c|c|c|c|}
\hline \multirow[t]{2}{*}{ Days after discharge } & \multicolumn{2}{|l|}{ Mortality, \% } & \multirow[b]{2}{*}{$P$ value } & \multicolumn{2}{|l|}{$\mathrm{HR}(95 \% \mathrm{Cl})$} \\
\hline & Non-sepsis $(n=142,646)$ & Sepsis $(n=1857)$ & & Unadjusted & Adjusted \\
\hline 30 days & 0.7 & 4.6 & $<0.001$ & $6.64(5.23-8.44)$ & $2.75(2.14-3.53)$ \\
\hline 60 days & 1.2 & 6.7 & $<0.001$ & $5.90(4.67-7.45)$ & $2.45(1.94-3.10)$ \\
\hline 90 days & 1.5 & 8.1 & $<0.001$ & $5.52(4.40-6.91)$ & $2.31(1.85-2.87)$ \\
\hline 1 year & 3.8 & 13.5 & $<0.001$ & $3.79(3.25-4.42)$ & $1.71(1.46-2.00)$ \\
\hline
\end{tabular}

Note: The adjusted HRs were derived from Poisson mixed models adjusted for age, sex, country of birth, marital status, severity of illness/co-morbidity, Socio-Economic Indexes for Areas (SEIFA), hospital location, peer hospital group, major surgical procedures and length of stay 


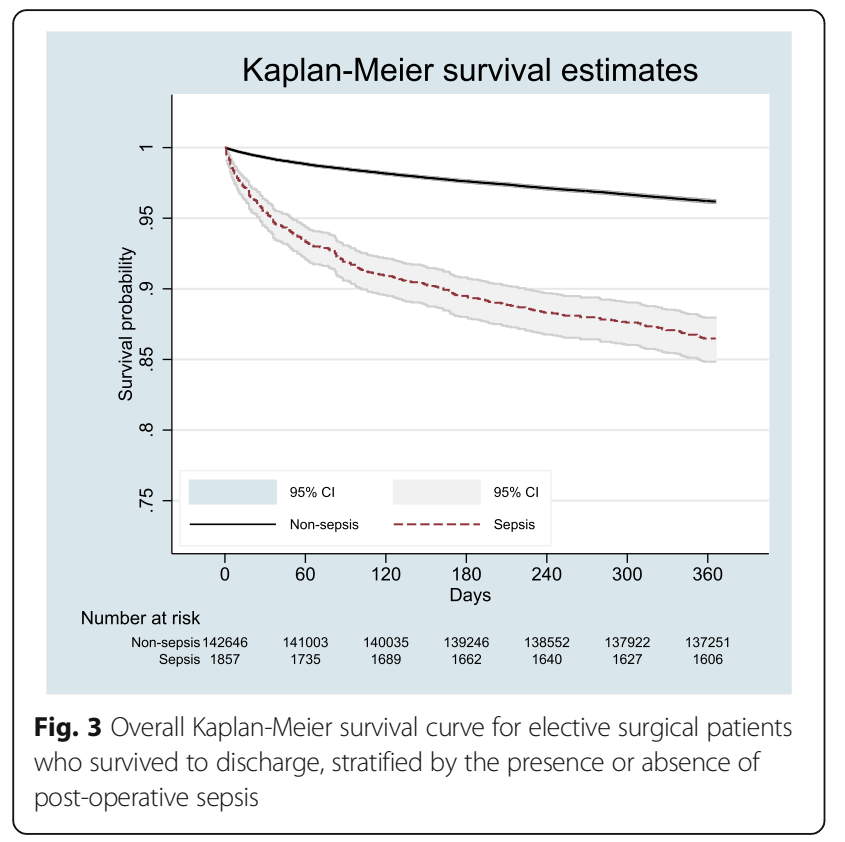

than that in the non-sepsis cohort (HR 2.04, 95\% CI $1.88-2.21)$.

\section{Discussion}

\section{Key findings}

We found that, among hospital survivors of elective surgery, the proportion experiencing post-operative sepsis increased 34\% over the study period, although overall elective surgical activity was stable. Importantly, we found that survivors who had experienced post-operative sepsis had substantially higher post-discharge 1 -year mortality (13.5\%) than patients without post-operative sepsis (3.8\%). This effect was more pronounced in the elderly, in those with co-morbidities and within the first 30 days postdischarge. Although age, sex, country of birth, marital status and co-morbidity strongly predicted the risk of long-term outcomes in the non-sepsis cohort, only advanced age and severe/very severe co-morbidities were independent predictors of 1-year mortality in the sepsis cohort. Finally, there was no significant change in 1-year
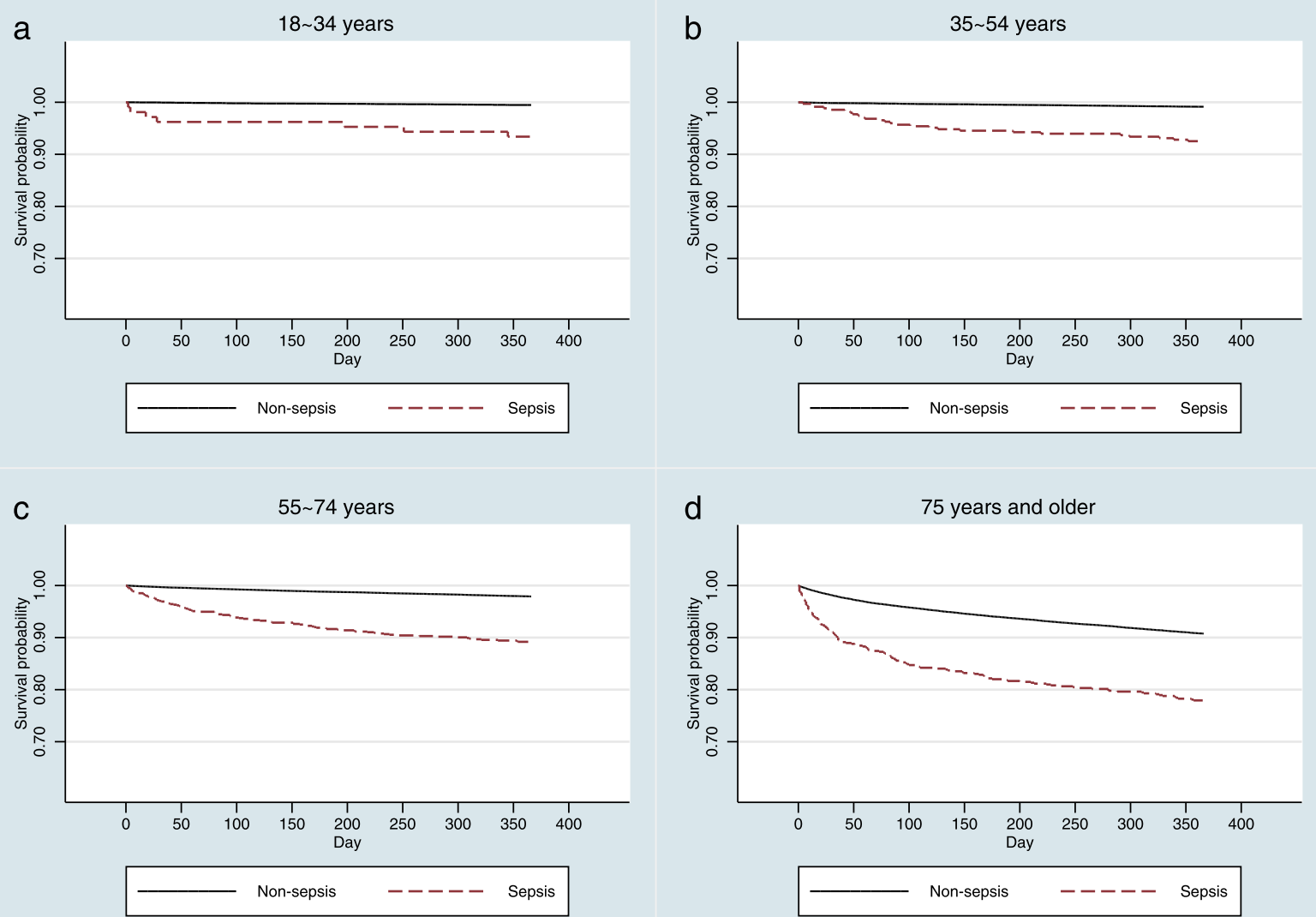

Fig. 4 Age-specific Kaplan-Meier survival curves for elective surgical patients who survived to discharge, stratified by the presence or absence of post-operative sepsis. a Patients aged 18-34 years. b Patients aged 35-54 years. c Patients aged 55-74 years. d Patients aged 75 years and older 
Table 3 Adjusted Cox proportional hazards models predicting 1-year post-discharge mortality

\begin{tabular}{|c|c|c|c|c|}
\hline \multirow[t]{2}{*}{ Characteristics } & \multicolumn{2}{|c|}{ Non-sepsis $(n=142,646)$} & \multicolumn{2}{|c|}{ Sepsis $(n=1857)$} \\
\hline & Mortality, \% & $\mathrm{HR}(95 \% \mathrm{Cl})$ & Mortality, \% & $\operatorname{HR}(95 \% \mathrm{Cl})$ \\
\hline \multicolumn{5}{|l|}{$\overline{\text { Year of admission }}^{\dagger}$} \\
\hline 2007 & 4.1 & 1.00 & 16.7 & 1.00 \\
\hline 2008 & 4.1 & $1.03(0.95-1.12)$ & 10.9 & $0.70(0.43-1.14)$ \\
\hline 2009 & 3.9 & $1.03(0.94-1.12)$ & 11.8 & $0.76(0.48-1.21)$ \\
\hline 2010 & 3.7 & $1.05(0.92-1.19)$ & 16.1 & $1.04(0.76-1.43)$ \\
\hline 2011 & 3.7 & $1.05(0.96-1.15)$ & 14 & $0.88(0.60-1.31)$ \\
\hline 2012 & 3.5 & $0.98(0.89-1.09)$ & 11.8 & $0.78(0.48-1.28)$ \\
\hline \multicolumn{5}{|l|}{ Age groups } \\
\hline$\geq 18$ years to $<35$ years & 0.5 & 1.00 & 6.6 & 1.00 \\
\hline$\geq 35$ years to $<55$ years & 0.9 & $1.99^{* *}(1.35-2.93)$ & 7.5 & $1.10(0.40-3.04)$ \\
\hline$\geq 55$ years to $<75$ years & 2.1 & $5.46^{* *}(3.77-7.92)$ & 10.8 & $1.60(0.76-3.38)$ \\
\hline$\geq 75$ years & 9.2 & $20.0^{* *}(13.5-29.6)$ & 22.1 & $3.50 * *(1.56-7.87)$ \\
\hline \multicolumn{5}{|l|}{ Sex } \\
\hline Male & 4.3 & 1.00 & 13.4 & 1.00 \\
\hline Female & 3.4 & $0.76^{* *}(0.71-0.82)$ & 13.7 & $1.05(0.81-1.37)$ \\
\hline \multicolumn{5}{|l|}{ Country of birth } \\
\hline Australia and New Zealand & 4.1 & 1.00 & 13.9 & 1.00 \\
\hline United Kingdom, United States and Canada & 4.4 & $0.96(0.88-1.05)$ & 11.7 & $0.66(0.36-1.19)$ \\
\hline Non-English-speaking Europe & 4.1 & $0.83^{* *}(0.74-0.93)$ & 17.3 & $0.97(0.69-1.36)$ \\
\hline North Africa & 1.5 & $0.50^{* *}(0.44-0.58)$ & 7.7 & $0.52(0.18-1.53)$ \\
\hline Asia & 1.9 & $0.68^{* *}(0.54-0.85)$ & 10.8 & $0.80(0.37-1.72)$ \\
\hline Others & 1.7 & $0.66^{* *}(0.53-0.83)$ & 6.5 & $0.47(0.18-1.21)$ \\
\hline Unknown & 5.6 & $1.02(0.81-1.28)$ & 17.2 & $1.45(0.76-2.78)$ \\
\hline \multicolumn{5}{|l|}{ Marital status } \\
\hline Married & 2.9 & 1.00 & 12.4 & 1.00 \\
\hline Single & 4.8 & $1.37^{* *}(1.28-1.46)$ & 14.9 & $1.10(0.89-1.36)$ \\
\hline Unknown & 6.4 & $1.67^{* *}(1.37-2.03)$ & 12.8 & $0.93(0.39-2.24)$ \\
\hline \multicolumn{5}{|l|}{ Severity of illness/co-morbidity } \\
\hline Normal & 2.5 & 1.00 & 9.2 & 1.00 \\
\hline Moderate & 7.5 & $2.04^{* *}(1.88-2.21)$ & 9.9 & $0.96(0.61-1.50)$ \\
\hline Severe & 10.1 & $2.34^{* *}(2.01-2.72)$ & 20.2 & $2.05^{* *}(1.45-2.89)$ \\
\hline Very severe & 15.5 & $3.34^{* *}(2.99-3.72)$ & 24.6 & $2.17^{* *}(1.49-3.17)$ \\
\hline \multicolumn{5}{|l|}{ SEIFA quartiles } \\
\hline First quartile (most disadvantaged) & 4.0 & 1.00 & 14.9 & 1.00 \\
\hline Second quartile & 3.7 & $0.93(0.85-1.00)$ & 13.2 & $0.91(0.65-1.26)$ \\
\hline Third quartile & 3.5 & $0.94(0.83-1.06)$ & 11.8 & $0.71 *(0.52-0.97)$ \\
\hline Fourth quartile (most advantaged) & 4.3 & $0.99(0.80-1.23)$ & 13.1 & $0.68(0.35-1.32)$ \\
\hline Unknown & 2.4 & $0.64^{*}(0.41-1.00)$ & 20.8 & $1.59(0.90-2.84)$ \\
\hline \multicolumn{5}{|l|}{ Local health district of facility } \\
\hline Metropolitan & 3.7 & 1.00 & 13.8 & 1.00 \\
\hline Rural and regional NSW & 4.3 & $1.06(0.88-1.27)$ & 12.6 & $0.81(0.60-1.10)$ \\
\hline Others & 3.1 & $0.72^{* *}(0.64-0.81)$ & 12.9 & $1.08(0.70-1.67)$ \\
\hline
\end{tabular}


Table 3 Adjusted Cox proportional hazards models predicting 1-year post-discharge mortality (Continued)

\begin{tabular}{|c|c|c|c|c|}
\hline \multicolumn{5}{|l|}{ Peer hospital group } \\
\hline Principal referral group & 3.9 & 1.00 & 12.4 & 1.00 \\
\hline Ungrouped acute & 2.2 & $0.59^{* *}(0.44-0.80)$ & 0 & - \\
\hline Major metropolitan and non-metropolitan & 4 & $1.20(1.00-1.45)$ & 17.1 & $1.53^{*}(1.10-2.11)$ \\
\hline District groups 1 and 2 & 2.7 & $1.10(0.85-1.44)$ & 12.2 & $1.04(0.40-2.73)$ \\
\hline \multicolumn{5}{|l|}{ Major surgical procedure } \\
\hline CABG & 2.0 & 1.00 & 7.0 & 1.00 \\
\hline Abdominal surgery & 2.8 & $1.91^{* *}(1.50-2.43)$ & 8.3 & $1.25(0.61-2.56)$ \\
\hline EVAR & 7.3 & $2.40^{* *}(1.92-3.00)$ & 25.0 & $3.79^{* *}(1.39-10.3)$ \\
\hline Total hip replacement & 1.5 & $0.80(0.62-1.03)$ & 11.1 & $1.51(0.47-4.78)$ \\
\hline Total knee replacement & 0.8 & $0.46^{* *}(0.36-0.59)$ & 5.3 & $0.68(0.19-2.42)$ \\
\hline Other & 6.1 & $3.13^{* *}(2.50-3.91)$ & 19.0 & $2.69 * *(1.38-5.22)$ \\
\hline Length of stay & & $1.01^{* *}(1.01-1.01)$ & & $1.00^{* *}(1.00-1.01)$ \\
\hline
\end{tabular}

post-discharge mortality for sepsis over the 6-year study period.

\section{Relationship to previous studies}

Previous studies have shown that post-discharge 1-year mortality in patients with the general diagnosis of sepsis ranged from $21.5 \%$ for those admitted through emergency departments [15] to $71.9 \%$ for those discharged from an ICU [17]. Such values are much higher than our reported rate of $13.5 \%[18,20]$. This is not surprising, because elective surgical patients have a much lower overall mortality [29]. However, our point estimate was in line with recent systematic review results (14.0-18.0\%) based on 43 studies [30].

Studies of long-term mortality related to sepsis in nonshort-stay patients having elective surgery are few and have been focused on either specific types of surgery such as endovascular surgery or open abdominal aneurysm repair [21], or patients with cancer having gastrointestinal surgery [22]. In contrast, we studied all elective surgical patients, thereby providing the first estimate of risk on the broader population having elective surgery. Moreover, we excluded patients with a principal diagnosis of sepsis or infection, as well as those with cancer or who were immunocompromised. Thus, our study population was more likely to reflect patients for whom post-operative sepsis was potentially preventable. Nonetheless, the reported post-hospital discharge 30-day and 90-day mortality for patients undergoing elective open surgery and endovascular repair of non-ruptured AAAs, using the AHRQ definition of post-operative sepsis, were similar to ours [21]. Finally, we identified post-operative sepsis on the basis of the AHRQ definition, which differs slightly from the definitions used in other studies. None of the 11 studies [8, 31-40] which used administrative data in studying sepsis and its outcomes adopted exactly the same definition and research questions as we did in our present study, which made explicit comparisons impossible.

Our study findings of consistently elevated risk of post-discharge mortality up to 1 year among the sepsis survivors compared with the non-sepsis cohort were in contrast with a recently published systematic review [30] in which the authors did not consistently observe a causal relationship between sepsis and post-discharge mortality. Such consistently elevated risks were presented according to three approaches adopted in our study: a multivariate Cox regression model, NNM and PSM. Of these approaches, NNM showed the most significant effect. The reasons why our study showed such a positive link may be due to the facts that our study sample included only elective surgical patients with specific inclusion criteria; that our study sample was more homogeneous and the control cohort was more comparable; and that our study included more recent data (January 2007 through December 2012) than most of the study data included in the review, which was prior to 2005. However, our study also showed results consistent with those of the review in that age and co-morbidities were independent predictors of mortality among sepsis survivors.

Our detailed analyses confirmed that the 1-year postdischarge mortality gap between septic and non-septic elective surgical patients was particularly pronounced among the elderly and among those patients with comorbidities [17, 41]. Researchers in previous studies reported similar in-hospital mortality rates between 
female and male surgical patients who developed severe sepsis or septic shock and those admitted to an ICU [42]. We also found similar 1-year post-discharge mortality rates between male and female patients in the septic cohort.

\section{Study implications}

Our study has several clinical and policy implications. The data show that more than one in eight non-shortstay surgical patients who develop post-operative septic patients will die within 1 year. Being elective hospital admissions, some of these deaths may be preventable. As older patients with significant co-morbidities appear to be more at risk, our study implies such patients warrant closer assessment and targeted strategies to reduce the risk of post-operative sepsis. Our study also implies that assessing the impact of post-operative sepsis using survival at hospital discharge is flawed [12, 43]. Our finding that the greatest rate of decline in survival is within the first 30-day period after discharge implies that this immediate post-discharge period has the greatest potential as the key time frame for intervention.

It is worth noting that the incidence of post-operative sepsis had increased while the case fatality declined over the 6-year (2007-2012 inclusive) period, showing that the overall incidence rate of sepsis-related deaths in hospital barely changed between 2009 and 2012. We cannot offer a definitive answer on whether such an increase in the sepsis incidence rate was due to better coding or a real rise in the incidence. However, our results also show that the 1-year post-discharge mortality among sepsis survivors did not change significantly over the same period. This may imply that the increased coding of sepsis is unlikely to be the case for the observed increased incidence rate because it is likely that increased coding practice may pick up mostly less severe sepsis cases. As a result, the increased incidence of sepsis will lead to an increased number of sepsis survivors in better health and reduced 1-year post-discharge mortality over that time. On the contrary, our results showed a flat 1-year post-discharge mortality over that time, suggesting that more patients with sepsis were discharged alive but died within 1 year (most likely within 30 days) during the study period. Such results highlight the importance of developing new policy initiatives in providing better coordinated care for these patients and managing this shifted care burden.

\section{Strengths and limitations}

This study has several strengths. To the best of our best knowledge, this is the largest population-based epidemiological study to provide evidence of an increased risk of post-discharge mortality for non-short-stay elective surgical patients with post-operative sepsis.
Our study is also the first to demonstrate a persistently high risk of death among those sepsis survivors over a 12 -month post-operative period. Our findings also identify more vulnerable patient subgroups, potentially providing clinical and policy-relevant information that could be used for benchmarking and translational interventions.

Our study also carries some limitations. First, we excluded patients with cancer and those who were immunologically compromised or those with a short hospital stay. In this study, we intended to target those patients whose sepsis was likely to be acquired through the exogenous factors which lent themselves to being more likely to be preventable. Moreover, these patients are also more likely to have benefited from the prompt clinical intervention and rescue, even when they developed sepsis during their hospitalization (e.g., the 'failure-to-rescue' definition of the AHRQ). However, we also acknowledge the fact that, in some cancer or immunocompromised patients who developed sepsis, their sepsis may also, in part, have been preventable, and they may have been rescued. Thus, we cannot comment on the incidence and implications of post-operative sepsis in such patients. However, our goal was to assess preventable post-operative sepsis in the presence of the smallest possible number of confounders and after excluding very low-risk patients. Second, despite the use of professional and certified coders to extract chart data, the absolute accuracy of such data extraction cannot be guaranteed. However, all large database analyses must depend on such coding, and the administrative data extracted by certified professional coders based on standardised guidelines at each hospital is unlikely to carry systematic bias.

Third, there may be a very small number of patients who were discharged to and died at another hospital, which could lead to an underestimation of in-hospital mortality. Despite the fact that we excluded those elective surgical patients who were principally diagnosed with sepsis or infection on admission, given the possibility of miscoding and misdiagnosis, further studies may be needed to provide an estimate of the extent that some of these septic cases may represent community-acquired sepsis. Furthermore, we did not have data on the time between surgery and post-operative sepsis events, surgical sites, and post-operative sepsis, and thus we could not make a clear differentiation between the surgical site infections vs non-surgical site infections among sepsis cohorts. Further designated studies are needed to explore these relationships. Finally, we did not have information on causes of death; thus, we could not relate the development of in-hospital sepsis with long-term mortality in any detail. In this regard, the development of sepsis after surgery may represent a causative factor or another marker of greater clinical fragility or both. 
Only interventional studies aimed at decreasing postoperative sepsis will provide a better understanding of the potential causative effect of post-operative sepsis on mortality.

\section{Conclusions}

In a large epidemiological study of non-short-stay elective surgical patients, we found that approximately 1 in 65 patients developed post-operative sepsis and that, among survivors, 1 in 8 died within 1 year after discharge. The first month post-discharge was the highest-risk period for mortality, and older patients and those with co-morbidities were those most at risk. Strategies that target the first month after discharge in this group of patients should be considered and evaluated.

\section{Additional files}

Additional file 1: Appendix 1. ICD-10-AM codes used to define postoperative sepsis. (DOCX $16 \mathrm{~kb}$ )

Additional file 2: Appendix 2. Codes used for exclusion criteria. (DOCX $104 \mathrm{~kb}$ )

Additional file 3: Appendix 3. Procedure codes from ICD-10-AM for selected surgical procedures. (DOCX $15 \mathrm{~kb}$ )

Additional file 4: Appendix 4. Results from sensitivity analyses. (DOCX $20 \mathrm{~kb}$ )

\section{Abbreviations}

AAA: Abdominal aortic aneurysm; AHRQ: Agency for Healthcare Research and Quality; APDC: Admitted Patient Data Collection; CABG: Coronary artery bypass graft; ED: Emergency department; EVAR: Endovascular aneurysm repair; ICD-9-CM: International Classification of Diseases, Ninth Revision, Clinical Modification; ICD-10-AM: International Statistical Classification of Diseases and Related Health Problems, Tenth Revision, Australian Modification; ICU: Intensive care unit; IR: Incidence rate of sepsis reported per 1000 admissions; LOS: Length of stay; MDC: Major Diagnostic Categories; NNM: Nearest-neighbour matching; NSW: New South Wales; OECD: Organisation for Economic Co-operation and Development; PSM: Propensity score matching; RBDM: New South Wales Registry of Births, Deaths, and Marriages; SEIFA: Socio-Economic Indexes for Areas

\section{Acknowledgements}

Not applicable.

\section{Funding}

Research funding for the study was provided by the National Health and Medical Research Council (NHMRC grants ID1020660 and ID1009916), Australia. The funding body played no role in the study design, conduct, analyses, or interpretation or in the writing of the study results.

\section{Availability of data and materials}

Not applicable.

\section{Authors' contributions}

$\mathrm{LO}$ and $\mathrm{JC}$ participated in the design of the study and the drafting of the manuscript. LO participated in data linkage, statistical analysis, interpretation of results, and initial manuscript drafting. JC, KH, AF, MP, RB, and HA critically revised the manuscript. All authors read and approved the final manuscript.

\section{Competing interests}

The authors declare that they have no competing interests.
Consent for publication

Not applicable.

Ethics approval and consent to participate

Not applicable.

\section{Author details}

${ }^{1}$ Simpson Centre for Health Services Research, South Western Sydney Clinical School, University of New South Wales, Sydney, New South Wales, Australia. ${ }^{2}$ Ingham Institute for Applied Medical Research, Liverpool, New South Wales, Australia. ${ }^{3}$ Intensive Care Unit, Royal Adelaide Hospital, Adelaide, South Australia, Australia. ${ }^{4}$ Faculty of Health Sciences, School of Medicine, University of Adelaide, Adelaide, South Australia, Australia. ${ }^{5}$ Intensive Care Unit, Liverpool Hospital, University of New South Wales, Sydney, New South Wales, Australia. ${ }^{6}$ Epidemiology and Health Analytics, South Western Sydney Local Health District, Sydney, New South Wales, Australia. ${ }^{7}$ School of Medicine, University of Melbourne, Parkville, Melbourne, Victoria, Australia.

Received: 20 July 2016 Accepted: 31 December 2016 Published online: 20 February 2017

\section{References}

1. Finks JF, Osborne NH, Birkmeyer JD. Trends in hospital volume and operative mortality for high-risk surgery. N Engl J Med. 2011;364(22):2128-37.

2. Leapfrog Hospital Survey. Factsheet: evidence-based hospital referral. http:// www.leapfroggroup.org/sites/default/files/Files/EBHR\%20Fact\%20Sheet.pdf. Accessed 8 May 2016.

3. Vogel TR, Dombrovskiy VY, Carson JL, Graham AM, Lowry SF. Postoperative sepsis in the United States. Ann Surg. 2010:252(6):1065-71.

4. Centers for Disease Control and Prevention (CDC). Healthcare-associated infections: HAl data and statistics. Atlanta, GA: CDC. http://www.cdc.gov/ $\mathrm{HAl} /$ surveillance/index.html. Accessed 16 May 2016.

5. Winters BD, Eberlein M, Leung J, Needham DM, Pronovost PJ, Sevransky JE. Long-term mortality and quality of life in sepsis: a systematic review. Crit Care Med. 2010;38(5):1276-83.

6. Yende S, Angus DC. Long-term outcomes from sepsis. Curr Infect Dis Rep. 2007;9(5):382-6.

7. Quartin AA, Schein RMH, Kett DH, Peduzzi PN. Magnitude and duration of the effect of sepsis on survival. JAMA. 1997;277(13):1058-63.

8. Weycker D, Akhras KS, Edelsberg J, Angus DC, Oster G. Long-term mortality and medical care charges in patients with severe sepsis. Crit Care Med. 2003;31(9):2316-23.

9. Prescott HC, Langa KM, Iwashyna TJ. Readmission diagnoses after hospitalization for severe sepsis and other acute medical conditions. JAMA. 2015;313(10):1055-7. doi:10.1001/jama.2015.1410.

10. Lee H, Doig CJ, Ghali WA, Donaldson C, Johnson D, Manns B. Detailed cost analysis of care for survivors of severe sepsis. Crit Care Med. 2004;32(4):981-5. doi:10.1097/01.CCM.0000120053.98734.2C.

11. Agency for Healthcare Research and Quality (AHRQ). AHRQ quality indicators: patient safety indicators: technical specifications. Version 3.2. Washington, DC: AHRQ; 10 Mar 2008. http://www.qualityindicators.ahrq.gov/ downloads/modules/psi/v32/psi_technical_specs_v32.pdf. Accessed 8 March 2016.

12. Bateman BT, Schmidt U, Berman MF, Bittner EA. Temporal trends in the epidemiology of severe postoperative sepsis after elective surgery: a large, nationwide sample. Anesthesiology. 2010;112(4):917-25.

13. Fried E, Weissman C, Sprung C. Postoperative sepsis. Curr Opin Crit Care. 2011;17(4):396-401.

14. Vogel TR, Dombrovskiy VY, Lowry SF. Impact of infectious complications after elective surgery on hospital readmission and late deaths in the U.S. Medicare population. Surg Infect (Larchmt). 2012;13(5):307-11. doi:10.1089/sur.2012.116.

15. Chen MJ, Tseng HM, Huang YL, Hsu WN, Yeh KW, Wu TL, et al. Long-term outcome and short-term survival of patients with systemic lupus erythematosus after bacteraemia episodes: 6-yr follow-up. Rheumatology (Oxford). 2008:47(9):1352-7. doi:10.1093/rheumatology/ken196.

16. Davis JS, He V, Anstey NM, Condon JR. Long term outcomes following hospital admission for sepsis using relative survival analysis: a prospective cohort study of 1,092 patients with 5 year follow up. PLoS One. 2014;9(12): e112224. 
17. Regazzoni CJ, Zamora RJ, Petrucci E, Pisarevsky AA, Saad AK, De Mollein D, et al. Hospital and 1-year outcomes of septic syndromes in older people: a cohort study. J Gerontol A Biol Sci Med Sci. 2008;63(2):210-2.

18. Shapiro NI, Howell MD, Talmor D, Donnino M, Ngo L, Bates DW. Mortality in Emergency Department Sepsis (MEDS) score predicts 1-year mortality. Crit Care Med. 2007:35(1):192-8. doi:10.1097/01.CCM.0000251508.12555.3E.

19. Linder A, Guh D, Boyd JH, Walley KR, Anis AH, Russell JA. Long-term (10year) mortality of younger previously healthy patients with severe sepsis/ septic shock is worse than that of patients with nonseptic critical illness and of the general population. Crit Care Med. 2014;42(10):2211-8.

20. Sasse KC, Nauenberg E, Long A, Anton B, Tucker HJ, Hu TW. Long-term survival after intensive care unit admission with sepsis. Crit Care Med. 1995; 23(6):1040-7. doi:10.1097/00003246-199506000-00008.

21. Faizer R, Dombrovskiy VY, Vogel TR. Impact of hospital-acquired infection on long-term outcomes after endovascular and open abdominal aortic aneurysm repair. Ann Vasc Surg. 2014;28(4):823-30.

22. Mokart D, Giaoui E, Barbier L, Lambert J, Sannini A, Chow-Chine L, et al. Postoperative sepsis in cancer patients undergoing major elective digestive surgery is associated with increased long-term mortality. J Crit Care. 2016; 31(1):48-53.

23. National Centre for Classification in Health (NCCH). The International Statistical Classification of Diseases and Related Health Problems, Tenth Revision, Australian Modification (ICD-10-AM). Sydney: NCCH, Faculty of Health Sciences, The University of Sydney; 2004.

24. Biondi N. Health Care Quality Indicators (HCQI) 2012-2013 data collection: technical manual for patient safety indicators. Paris: Organisation for Economic Co-operation and Development; 2012. http://www.oecd.org/els/ health-systems/20_Biondi_HCQIDataCollection.pdf. Accessed 10 Mar 2016.

25. Australian Bureau of Statistics. Census of population and housing: SocioEconomic Indexes for Areas (SEIFA), Australia, 2011. Canberra: Australian Bureau of Statistics; 2011

26. Quan H, Sundararajan V, Halfon P, Fong A, Burnand B, Luthi JC, et al. Coding algorithms for defining comorbidities in ICD-9-CM and ICD-10 administrative data. Med Care. 2005;43(11):1130-9.

27. NSW Health. NSW health services comparison data book 2008/2009. Sydney: NSW Health; 2010

28. King G, Nielsen R. Why propensity scores should not be used for matching. http://gking.harvard.edu/files/gking/files/psnot.pdf. Accessed 12 Dec 2016.

29. Heeney A, Hand F, Bates J, McCormack O, Mealy K. Surgical mortality - an analysis of all deaths within a general surgical department. Surgeon. 2014; 12(3):121-8.

30. Shankar-Hari M, Ambler M, Mahalingasivam V, Jones A, Rowan K, Rubenfeld GD. Evidence for a causal link between sepsis and long-term mortality: a systematic review of epidemiologic studies. Crit Care. 2016;20:101. doi:10.1186/s13054-016-1276-7.

31. Prescott HC, Osterholzer JJ, Langa KM, Angus DC, Iwashyna TJ. Late mortality after sepsis: propensity matched cohort study. BMJ. 2016;353:i2375 doi:10.1136/bmj.i2375

32. Yende S, Linde-Zwirble W, Mayr F, Weissfeld LA, Reis S, Angus DC. Risk of cardiovascular events in survivors of severe sepsis. Am J Respir Crit Care Med. 2014;189(9):1065-74. doi:10.1164/rccm.201307-13210C.

33. Prescott HC, Langa KM, Liu V, Escobar GJ, Iwashyna TJ. Increased 1-year healthcare use in survivors of severe sepsis. Am J Respir Crit Care Med. 2014;190(1):62-9. doi:10.1164/rccm.201403-04710C.

34. Ou SY, Chu H, Chao PW, Ou SM, Lee YJ, Kuo SC, et al. Effect of the use of low and high potency statins and sepsis outcomes. Intensive Care Med. 2014;40(10):1509-17.

35. Liu V, Lei X, Prescott HC, Kipnis P, Iwashyna TJ, Escobar GJ. Hospital readmission and healthcare utilization following sepsis in community settings. J Hosp Med. 2014;9(8):502-7. doi:10.1002/jhm.2197.

36. Chao PW, Shih CJ, Lee YJ, Tseng CM, Kuo SC, Shih YN, et al. Association of postdischarge rehabilitation with mortality in intensive care unit survivors of sepsis. Am J Respir Crit Care Med. 2014;190(9):1003-11. doi:10.1164/rccm. 201406-11700C

37. Iwashyna TJ, Netzer G, Langa KM, Cigolle C. Spurious inferences about longterm outcomes: the case of severe sepsis and geriatric conditions. Am J Respir Crit Care Med. 2012;185(8):835-41. doi:10.1164/rccm.201109-16600C.

38. Braun L, Riedel AA, Cooper LM. Severe sepsis in managed care: analysis of incidence, one-year mortality, and associated costs of care. J Manag Care Pharm. 2004;10(6):521-30.
39. Iwashyna TJ, Cooke CR, Wunsch H, Kahn JM. Population burden of longterm survivorship after severe sepsis in older Americans. J Am Geriatr Soc. 2012;60(6):1070-7. doi:10.1111/j.1532-5415.2012.03989.x.

40. Dick A, Liu H, Zwanziger J, Perencevich E, Furuya EY, Larson E, et al. Longterm survival and healthcare utilization outcomes attributable to sepsis and pneumonia. BMC Health Serv Res. 2012;12:432. doi:10.1186/1472-6963-12-432.

41. Ho KM, Knuiman M, Finn J, Webb SA. Estimating long-term survival of critically ill patients: the PREDICT model. PLoS One. 2008;3(9):e3226. doi:10.1371/journal.pone.0003226.

42. Wichmann MW, Inthorn D, Andress HJ, Schildberg FW. Incidence and mortality of severe sepsis in surgical intensive care patients: the influence of patient gender on disease process and outcome. Intensive Care Med. 2000; 26(2):167-72.

43. Kaukonen KM, Bailey M, Suzuki S, Pilcher D, Bellomo R. Mortality related to severe sepsis and septic shock among critically ill patients in Australia and New Zealand, 2000-2012. JAMA. 2014;311(13):1308-16. doi:10.1001/jama.2014.2637.

\section{Submit your next manuscript to BioMed Central and we will help you at every step:}

- We accept pre-submission inquiries

- Our selector tool helps you to find the most relevant journal

- We provide round the clock customer support

- Convenient online submission

- Thorough peer review

- Inclusion in PubMed and all major indexing services

- Maximum visibility for your research

Submit your manuscript at www.biomedcentral.com/submit
) Biomed Central 